\title{
INTERVENÇÃO FARMACÊUTICA NO PROCESSO DE CUIDADO FARMACÊUTICO: UMA REVISÃO
}

\section{The action of pharmacist in process of pharmacist-intervention: one review}

\author{
Mônica F. Z. J. Amaral ${ }^{1}$; Rita G. Amaral ${ }^{2}$; Mércia P. Provin². \\ ${ }^{1}$ Aluna de Graduação da Faculdade de Farmácia da UFG. Praça Universitária s/n. 74605-220. Goiânia-GO, Brasil \\ ${ }^{2}$ Professora da Faculdade de Farmácia, Universidade Federal de Goiás.
}

Autor para correspondência e.mail: merciap@gmail.com

Recebido em 12/03/2008 - Aceito em 10/06/2008

\begin{abstract}
RESUMO: Atenção Farmacêutica surge no cenário brasileiro e internacional como um novo exercício profissional onde o farmacêutico assume a responsabilidade de garantir que o paciente, que recebe um medicamento, possa cumprir os esquemas farmacoterapêuticos e seguir o plano de assistência, de forma a alcançar resultados positivos. Ao farmacêutico moderno é indispensável conhecimentos, atitudes e destrezas que permitam ao mesmo integrar-se à equipe de saúde e interagir mais com o paciente e a comunidade, contribuindo para a melhoria da qualidade de vida no que se refere à otimização da farmacoterapia e o uso racional de medicamentos. As ações do farmacêutico, no modelo de atenção farmacêutica, em sua grande maioria, são atos clínicos individuais. Mas as sistematizações das intervenções farmacêuticas e a troca de informações dentro de um sistema de informação composto por outros profissionais de saúde pode contribuir para um impacto em nível coletivo e na promoção do uso seguro e racional de medicamentos.
\end{abstract}

PALAVRAS-CHAVE: atenção farmacêutica, assistência farmacêutica, intervenções farmacêuticas.

ABSTRACT: Pharmaceutical care appears in Brazilian and international scene as a new professional practice where the pharmacist takes responsibility to ensure that the patient compliance whit your drug therapy and the plan of assistance to achieve positive outcome. In modern pharmacist is essential knowledge, attitudes and skill to allow even integrate to the team of health and interact more with patients and the community, contributing to improved quality of life regarding the optimization of pharmacotherapy and rational use of medicines. Shares of pharmacist, in the model of pharmaceutical care, in their vast majority, are clinical individual acts. But the systematization of pharmaceutical interventions and exchange of information within an information system composed of other health care professionals can contribute to an impact on collective level and promote the safe and rational use of medicines.

KEYWORD: pharmaceutical care, pharmaceutical practice, pharmaceutical intervention.

\section{INTRODUÇÃO}

Desde o surgimento do primeiro estabelecimento farmacêutico, até os dias atuais, o símbolo da farmácia é representado por uma taça com uma serpente entrelaçada, projetando-se do pé até o topo. A taça evoca o poder curativo das drogas, e a serpente, insinua uma atitude de advertência, ou seja, refere-se à cautela e responsabilidade ética com que deve atuar o farmacêutico.

No início do século XX, o farmacêutico era considerado pela sociedade, o profissional de referência quando o assunto era medicamento, atuando e exercendo influência sobre todas as fases do ciclo do medicamento. Neste período, além da posse e dispensação do medicamento, este era responsável pela produção e manipulação de todo o arsenal, disponível á população, daquela época. (GOUVEIA, 1999).

Na década de 50, com o crescimento da indústria farmacêutica e a diversificação do campo de atuação do profissional farmacêutico, houve um distanciamento deste profissional da área de medicamentos descaracterizando a farmácia, tornando a prática farmacêutica em uma simples distribuição de medicamentos industrializados (HEPLER \& GRAINGER-ROUSSEAU, 1995; GOUVEIA, 1999). 
Amaral, M. F. Z. J. et al./Revista Eletrônica de Farmácia Vol 5(1), 60-66, 2008.

Com o advindo da década de 60, ocorreu um aumento da insatisfação com essa nova forma de atuação decorrente do constante processo de industrialização, nascendo assim, um movimento profissional norte americano, que questionando a formação e as ações criou mecanismos para corrigir os problemas e permitir que os farmacêuticos participassem da equipe de saúde usando seus conhecimentos para melhorar o cuidado ao paciente. O resultado foi o surgimento no âmbito hospitalar da farmácia clínica, onde o profissional tornou-se um consultor farmacoterapêutico. (HOLLAND \& NIMMO, 1999; HEPLER, 2004).

$\mathrm{Na}$ década de 1990, Hepler \& Strand sugerem uma nova atividade profissional ao farmacêutico, com o objetivo de reduzir a morbilidade e mortalidade associada aos medicamentos. Este novo exercício profissional foi denominado de Pharmaceutical Care, traduzido para Atenção Farmacêutica, em português (HEPLER \& STRAND, 1990).

Segundo Hepler e Strand, a definição clássica de Atenção Farmacêutica baseia-se na dispensa responsável da terapêutica farmacológica com o objetivo de alcançar resultados definitivos que contribuam para a melhoria da qualidade de vida do doente (HEPLER \& STRAND,1990).

$\mathrm{Na}$ atenção farmacêutica o farmacêutico passa a atuar de forma mais efetiva na assistência ao paciente. $\mathrm{O}$ profissional se responsabiliza pela necessidade, segurança e efetividade da farmacoterapia do paciente. Isto se consegue mediante a identificação, resolução e prevenção dos problemas relacionados com medicamentos (OPAS, 2002b). Ao prestar atenção farmacêutica o profissional se responsabiliza de garantir que o paciente possa cumprir os esquemas farmacoterápicos e seguir o plano de assistência, de forma a alcançar resultados positivos (LEE \& RAY, 1993). Deste modo a farmacoterapia se converte na forma de intervenção farmacêutica mais empregada (LYRA et al, 2000; HEPLER \& GRAINGER-ROUSSEAU, 1994).

O conceito de intervenção farmacêutica é usado para denominar todas as ações da qual o farmacêutico participa ativamente como nas tomadas de decisão, na terapia dos pacientes e também na avaliação dos resultados. Torna-se imprescindível para o farmacêutico ter a noção exata de sua competência, e dos limites de sua intervenção no processo saúde-doença (ZUBIOLI, 2000).

Ao farmacêutico moderno é indispensável conhecimentos, atitudes e destrezas que permitam ao mesmo integrar-se à equipe de saúde e interagir mais com o paciente e a comunidade, contribuindo para a melhoria da qualidade de vida no que se refere à otimização da farmacoterapia e o uso racional de medicamentos (OPAS, 2003)

As ações do farmacêutico, no modelo de atenção farmacêutica, em sua grande maioria, são atos clínicos individuais. Mas as sistematizações das intervenções farmacêuticas e a troca de informações dentro de um sistema de informação composto por outros profissionais de saúde pode contribuir para um impacto em nível coletivo e na promoção do uso seguro e racional de medicamentos. (OPAS, 2002c).

De acordo com o exposto acima, este artigo faz uma breve revisão de todo o processo de intervenção farmacêutica, desde sua metodologia até exposição das publicações mais recentes neste assunto, avaliando deste modo a grande importância do profissional farmacêutico na farmacoterapia.

\section{Metodologia da implementação da Intervenção Farmacêutica}

A intervenção farmacêutica ocorre quando o profissional realiza a monitorização farmacoterapêutica. Monitorização farmacoterapêutica é um processo contínuo, que identifica e resolve problemas relacionados com os medicamentos. Diante disto o farmacêutico pode realizar intervenções visando aumentar a efetividade e diminuir os riscos da farmacoterapia. Na monitorização farmacoterapêutica são avaliados (KEITH \& FOSTER, 1992; FARRÉ et al 2000):

- A veracidade do regime farmacoterapêutico direcionado ao paciente;

- Sobreposições terapêuticas;

- Veracidade da via e método de administração do fármaco;

- Aceitação do paciente a terapia prescrita;

- Interações do medicamento com outros fármacos, com alimento, ou com alguma patologia;

- Dados clínicos e estatísticos para avaliar a eficácia da farmacoterapia e antecipar os efeitos adversos, toxicidade e os sinais clínicos que são relevantes para a farmacoterapia que recebe o paciente.

Para que isto ocorra de uma forma otimizada, o processo de monitorização terapêutica deve ser dividido em etapas, na qual facilite a avaliação no processo de intervenção do farmacêutico.

\section{Triagem dos pacientes}

A realização de uma seleção de grupos de pacientes é imprescindível, já que não é possível analisar todos os grupos. Para tanto se torna extremamente necessário escalonar os pacientes que se encontram em um grupo de maior risco para o desenvolvimento de problemas relacionados aos medicamentos.

A seleção dos pacientes pode ser dividida em 2 grandes grupos: Características individuais do paciente e característica dos medicamentos utilizados (GRANDA, 2004; OPAS, 2003).

$\mathrm{Na}$ análise das características individuais dos pacientes, podemos avaliar os seguintes critérios: faixa etária (Crianças e idosos, por exemplo) e patologia (diabetes, hipertensão, enfermidades cardíacas, imunossuprimidos, enfermidades hepáticas, dentre outros). Na análise das características dos medicamentos, podemos considerar critérios como: quantidade de medicamentos utilizados, pacientes que utilizam fármacos com 
alto risco de toxicidade (anticonvulsivantes e antiarrítmicos) e pacientes que utilizam mais de um fármaco para a mesma patologia (diabéticos, hipertensos) (FARRÉ et al, 2000; OPAS, 2003).

\section{Análise dos dados}

Esta etapa da análise é essencial para se obter bons resultados na monitorização terapêutica. As informações pertinentes devem ser obtidas através da história clinica do paciente, falando diretamente com este ou seus familiares, ou ainda buscando informações adicionais com os outros profissionais da saúde responsáveis por este (CANADAY \& YARBOROUGH, 1994).

Em resumo os dados a serem reunidos são (FARRÉ et al, 2000):

- Informações gerais sobre o paciente (sexo, idade, peso, altura, dentre outros);

- Casos de patologias crônicas ou neoplasias no histórico familiar;

- Diagnóstico da patologia;

- Alergias;

- Hábitos (consumo de álcool e cigarro)

- Dieta alimentar;

- Cumprimento do tratamento prescrito;

- Exames laboratoriais;

- Medicamentos utilizados;

Um modo de se obter tais informações de forma padronizada é utilizando modelos de formulários, nas quais todas as informações anteriores são incluídas, facilitando o processo de unificação dos dados (OPAS, 2003; LAL et al, 1995).

A freqüência com que são realizadas tais entrevistas depende da situação especifica de cada paciente, nos quais alguns requerem monitorização semanal, outros quinzenais ou até mensais.

\section{Detecção dos problemas}

Realizada a análise, a informação obtida pode ou não gerar uma lista de problemas farmacoterapêuticos do paciente. Para tanto são avaliadas questões como (FARRÉ et al, 2000):

- Se há duplicidade na terapia;

- Se a posologia é adequada;

- Se o medicamento selecionado é o ideal;

- Se há alergias e intolerância ao medicamento;

- Avaliações das reações adversas;

- Se há interações;

- Se há ou não cumprimento da terapia;

- E se há uma boa relação custo - beneficio na terapia;

Discussão da intervenção entre os profissionais da saúde

Detectado o problema, e proposto uma intervenção para este, há a necessidade de comunicá-la a equipe de profissionais responsáveis pelo paciente. Esta comunicação pode ser telefônica, através de reuniões, ou escrita (OPAS, 2003; FARRÉ et al, 2000).

Destas vias, a que se obtém maior eficiência na comunicação, é através de reuniões, onde os profissionais podem discutir abertamente e analisar juntos o processo de intervenção como um todo.

\section{Documentações das intervenções}

As decisões tomadas pelo profissional farmacêutico devem ser documentadas, a fim de garantir uma boa reprodutibilidade do processo. O modelo de documentação a ser escolhido é aquele que melhor se adapta a forma de trabalho do profissional, tendo que ser completo e de rápido acesso (LAL et al, 1995). Então cabe ao farmacêutico personalizar seu próprio sistema de documentação.

\section{Classificação das intervenções}

Através de um simples questionário realizado pelo próprio farmacêutico, pode-se obter um código para a intervenção terapêutica analisada, que facilita o processo de análise do profissional, além de proporcionar uma otimização para o registro desta. De acordo com Farré et al (2000), as intervenções podem ser divididas em 6 grandes tipos (Quadro 1).

\section{Avaliação dos resultados das intervenções farmacêuticas}

Realizadas as intervenções, estas devem ser analisadas, avaliando os resultados obtidos com a aceitação da terapia pelo paciente. Farré et al e colaboradores (2000) conseguiram elaborar um sistema prático e de fácil 
Amaral, M. F. Z. J. et al./Revista Eletrônica de Farmácia Vol 5(1), 60-66, 2008.

manuseio, sem provocar aumento exagerado da carga de trabalho do profissional. De acordo com os autores a avaliação do impacto das intervenções pode ser realizada utilizando dois códigos principais: código de impacto e código de significação (Quadro 2).

O objetivo do código de impacto é medir quantitativamente se o processo de intervenção provocou um aumento da eficácia, ou uma diminuição da toxicidade na terapia medicamentosa.

Por outro lado o código de significação busca medir a atenção farmacoterapêutica prestada ao paciente e a sua contribuição na melhoria da qualidade, através de uma escala bem definida. Assim os autores dividem a escala em aspectos positivos e negativos, analisando se a intervenções foram apropriadas, indiferentes ou inapropriadas para o paciente em questão.

Quadro 1: Tipos de intervenções

\begin{tabular}{|c|l|}
\hline Tipos de intervenção & \multicolumn{1}{c|}{ Questionário } \\
\hline \multirow{2}{*}{ POSOLOGIA } & É a dose mais correta para este paciente? \\
& A freqüência entre as administrações é adequada? \\
& Por qual via o fármaco é eliminado? \\
& Há necessidade de reajuste de dose? \\
& A terapia pode ser encurtada? \\
\hline VIA DE ADMINISTRAÇÃO & É a via de administração mais adequada? \\
& Está sendo administrado corretamente? \\
\hline \multirow{2}{*}{ INDICAÇÃO } & A terapia é necessária? \\
& Esta sendo efetiva? \\
& Há alguma indicação que não está sendo tratada? \\
& A indicação convém com a patologia? \\
& Há duplicidade? \\
& Que parâmetros são úteis para monitorar a eficácia? \\
& Há um beneficio custo - efetividade? \\
\hline INTERAÇÃO & Interage com outros fármacos utilizados? \\
& Interage com algum alimento da dieta do paciente? \\
\hline \multirow{2}{*}{ EFEITOS ADVERSOS } & Este fármaco é o mais seguro para este paciente? \\
& Qual a gravidade dos efeitos adversos? \\
& O paciente apresenta alguma alergia? \\
\hline OUTROS & Outras perguntas? \\
\hline
\end{tabular}

Adaptado de Farré et al, 2000.

Quadro 2 . Código de impacto e significação das intervenções farmacêuticas.

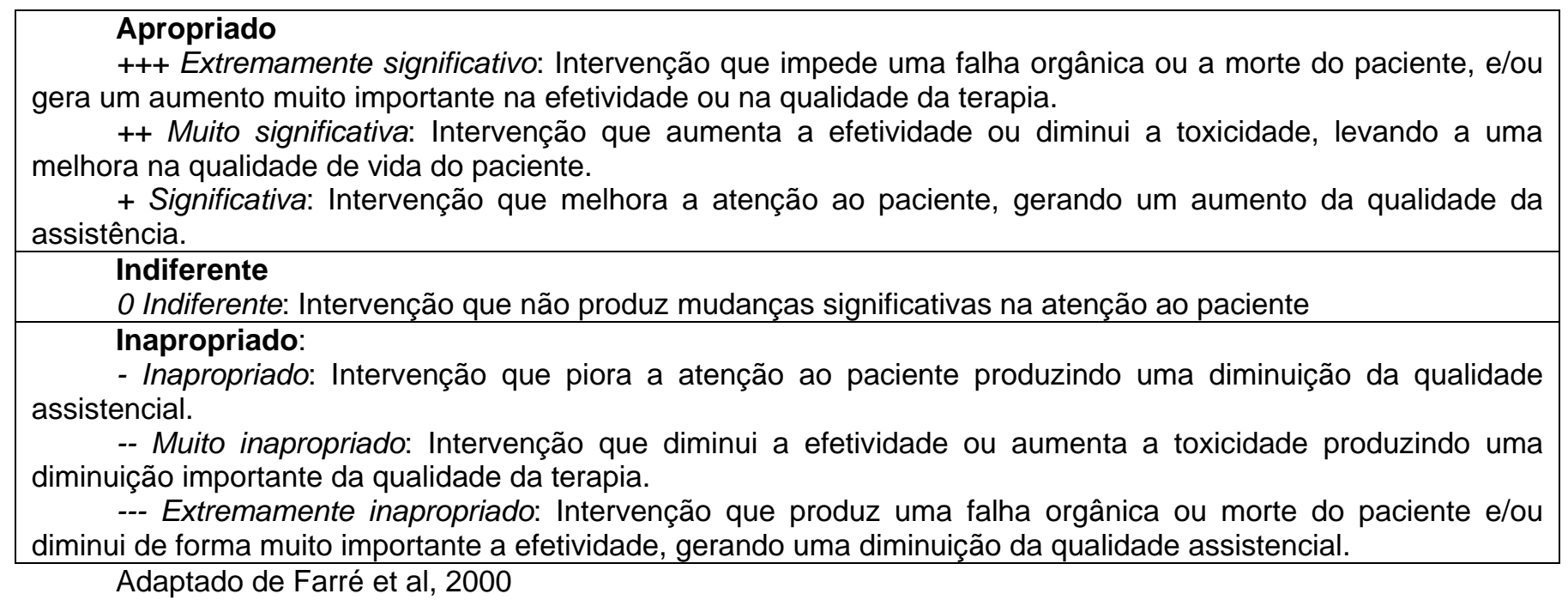

Apesar da aplicabilidade da avaliação acima citada, a metodologia ideal para se analisar as intervenções é o ensaio clínico, onde parâmetros humanísticos, clínicos e econômicos podem ser ponderados. Entretanto é de difícil execução no dia-a-dia do profissional (LIPOWSKI, 1996; PICKARD et al, 1999). 
Amaral, M. F. Z. J. et al./Revista Eletrônica de Farmácia Vol 5(1), 60-66, 2008.

O primeiro estudo importante sobre o impacto das ações de atenção farmacêutica foi realizado nos Estados Unidos utilizando os dados do Projeto Minnesota de Atenção Farmacêutica. Os resultados mostraram que após um ano aumentou o número de pacientes que alcançaram resultado terapêutico positivo. A resolução dos problemas relacionados a medicamentos reduziu a complexidade da demanda farmacoterápica. Em síntese observou uma relação custo-benefício favorável (PHARMACEUTICAL,1997).

A melhoria da qualidade de vida em pacientes com insuficiência cardíaca, diabetes, hipertensão e dislipidemia em virtude da intervenção farmacêutica têm sido demonstradas em vários estudos (MORSE et al, 1986; BARRÓN RIVERA et al, 1998; ARÁUZ et al, 2001; ERICKSON et al, 1997; CARRÍON et al, 2007; RAINVILLE et al, 1999; COWPER et al, 1998).

Weidle et al., descreveram as ações de intervenção farmacêutica de um programa de atenção farmacêutica em um hospital universitário. No hospital estudado $90 \%$ das ações estavam diretamente relacionado com o processo terapêutico e $10 \%$ com a otimização do custo assistencial. Os autores desenvolveram uma classificação com 11 categorias de intervenção farmacêuticas no âmbito hospitalar e as respectivas ações a serem implementadas (WEIDLE et al, 1999).

Romano-Lieber et al através de uma revisão bibliográfica, analisaram os estudos de intervenção farmacêutica realizados no período de 1970 a 1999 e sua influência no uso de medicamentos pelo paciente idoso, constatando que de um modo geral as intervenções mostravam resultados positivos, reduzindo custos, melhorando prescrições, promovendo maior adesão do paciente ao tratamento e controlando a possibilidade de reações adversas. Entretanto verificaram que a maioria das intervenções limitava-se ao aconselhamento ao usuário e/ou prescritor, notando-se que a falta de ações levavam a uma inadequação do medicamento ao usuário (ROMANO-LIEBER et al, 2002).

Company e colaboradores publicaram em 1998 um estudo realizado no Hospital Universitário del Mar de Barcelona, onde analisaram as intervenções farmacêuticas realizadas neste, através de um sistema informatizado de dispensação na dose unitária (COMPANY et al, 1998).

Outro estudo que demonstra claramente o aspecto positivo da intervenção farmacêutica foi realizado por Martínez e sua equipe, que avaliaram o impacto econômico e clinico da intervenção farmacêutica sobre a conversão da clindamicina intravenosa pela oral, em um grupo de 473 pacientes do Meixoeiro Hospital, da Espanha (MARTíNEZ et al, 2000). Uma pesquisa semelhante foi realizada por Santora et al, que analisaram um programa de intervenção farmacêutica centrada no uso da terapia de ranitidina intravenosa, demonstrando que um simples programa de intervenção e vigilância por parte dos profissionais farmacêuticos pode poupar dinheiro e promover uma adequada utilização do medicamento (SANTORA et al, 1990).

Os estudos citados acima evidenciam um impacto favorável da intervenção farmacêutica sobre a efetividade, qualidade de vida e custos assistenciais, sendo de grande valia a realização e publicação de pesquisas bem delineadas que analise a relevância da intervenção farmacêutica para os sistemas de saúde.

\section{CONCLUSÃO}

Um dos desafios da categoria farmacêutica é modificar as condutas, incorporando na prática profissional um modelo que propicie ao farmacêutico assumir a responsabilidade com a farmacoterapia. Para tanto a relação contínua entre paciente e farmacêutico é fundamental para que os serviços de intervenção farmacêutica sejam realizados de maneira ética e legal, fornecendo resultados permanentes assegurando a efetividade da terapia estabelecida.

Deste modo cabe aos profissionais farmacêuticos buscarem seus lugares frente às equipes de saúde, criando assim laços que sustente a sua prática profissional, indo ao encontro dos objetivos estabelecidos para a intervenção farmacêutica e procurando identificar e servir as necessidades reais do cidadão.

\section{REFERÊNCIAS BIBLIOGRÁFICAS}

ARÁUZ, A. G.; SÁNCHEZ, G.; PADILLA, G.; et al. Intervención educativa comunitaria sobre la diabetes en el âmbito de la atención primaria. Rev Panam Salud Publica/Pan Am J Public Health. v. 9, n. 3, p. 145-153, 2001.

BARRÓN-RIVERA, A. J.; TORREBLANCA-ROLDÁN, F. L.; SÁNCHEZ-CASANOVA, L. I.; et al. Efecto de uma intervención educativa em la calidad de vida del paciente hipertenso. Salud Publica Mex. v. 40, n. 3, p. 503509,1998 .

CANADAY, B. R.; YARBOROUGH, P. C. Documenting Pharmaceutical Care: creating a standard. The Annals of Pharmacotherapy . v. 28, n.11, p. 1292-1296, 1994.

CARRIÓN, C. C.; MONDEJAR, M. R. D. Efecto de uma intervención del tipo entrevista farmacoterapêutica sobre la prescripción de estatinas en atención primaria. Farmacia de Atención Primaria. v. 5, n.1, p. 8-14, 2007. 
Amaral, M. F. Z. J. et al./Revista Eletrônica de Farmácia Vol 5(1), 60-66, 2008.

COMPANY, D.; GRAU, S.; MONTERDE, J.; et al. Análisis de las intervenciones farmacéuticas realizadas a través del sistema informatizado de dispensación en dosis unitária. Farm Hosp. v. 22, n.1, p. 11-15, 1998.

COWPER, P. A.; WEINBERGER, M.; HANLON, J. T.; et al. The cost effectiveness of a clinical pharmacist intervention among elderly outpatients. Pharmacotherapy. v. 18, n .3, p. 327-332, 1998.

ERICKSON, S. R.; SLAUGHTER, R;. HALAPY, H. Pharmacists' ability to influence outcomes of hypertension therapy. Pharmacotherapy. v. 17, n. 2, p. 140-147, 1997.

FARRÉ, R.; CLOPÉS, A.; SALA, M. L.; et al. Intervenciones farmacêuticas (parte I): metodología y evaluación. Farm Hosp. v. 24, n. 3, p. 136-144, 2000.

GoUVEIA, W. A. At center stage: Pharmacy in the next century. Am. J. Health-Syst Pharm. v.56, p.2533, 1999.

HEPLER GRANDA, S. A. Evaluación de una intervención farmacêutica. Âmbito Farmacêutico: Farmacoeconomia. v. 23, n. 10, p. 112-119, 2004.

HEPLER, C. D.; STRAND, L. M. Opportunities and responsibilities in pharmaceutical care. American Journal of Hospital Pharmacy, v. 47, n. 3, p. 533-543, 1990.

HEPLER, C. D. The third wave in pharmaceutical education and clinical movement. Am J Pharm Educ. v. 51, n. 4, p. 369-385.

HEPLER, C. D.; GRAINGER-ROUSSEAU, T. J.; Pharmaceutical care versus traditional drug treatment. Is there a diference? Drugs. v. 49, n. 1, p. 1-10, 1995.

HOLLAND, R. W.; NIMMO, C. M. Transitions, part 1: Beyond pharmaceutical care. Am. J. Health-Pharm. v. 56, n.17, p. 1758-1764, 1999.

KEITH, T. D.; FOSTER, J. R. Drug Therapy Monitoring. Handbook of Institutional Pharmacy Practice, $3^{\mathrm{a}}$ ed. Ed. Brown TR. ASHP 1992; 273-8.

LAL, L. S.; ANASSI, E .O.; MCCANTS, E. Documentation of the First Steps of Pediatric Pharmaceutical Care in a County Hospital. Hosp Pharm. v. 30, n.12, p. 1107-1112,1995.

LEE, M. P.; RAY, M. D. Planning for pharmaceutical care. Am J. Hosp. Pharm. v. 50, n. 6, p. 1153-1158, 1993.

LIPOWSKI, E. E. Evaluating the Outcomes of Pharmaceutical Care. Journal of the American Pharmaceutical Association. v. NS36, n. 12, p. 726-736, 1996.

LYRA, J. R.; DIVALDO, P.; et al. Atenção farmacêutica: paradigma de globalização. Pharmácia Brasileira. v. 3, n. 23, p. 76-79, 2000.

MARTÍNEZ, M. J.; CASTRO, I.; INARAJA, M. T.; et al. Clinical and economic impact of a pharmacist-intervention to promote sequential intervention intravenous to oral clindamycin conversion. Pharmacy Wold and Science. v. 22, n. 1,p. 53-58, 2000.

MORSE, G. D.; DOUGLAS, J. B.; UPTON, J. H.; et al. Effect of Pharmacist intervention on control of resistant hypertension. Am J Hosp Pharm. v. 42, n. 4, p. 905-909, 1986.

ORGANIZAÇÃO PANAMERICANA DE SAÚDE. Consenso Brasileiro de Atenção Farmacêutica: Proposta. Brasília, OPAS, 24 p, 2002b.

ORGANIZAÇÃO PANAMERICANA DE SAÚDE. Termo de Referência para reunião do grupo de trabalho: Interface entre Atenção Farmacêutica e Farmacovigilância. Brasília, OPAS, 28 p, 2002c.

ORGANIZAÇÃO PANAMERICANA DE SAÚDE. Assistência Farmacêutica para gerentes municipais. Brasília, OPAS, 375p, 2003.

PHARMACEUTICAL Care: The minnesota model. Pharm. J. v. 258, n. 6949, p. 899-904, 1997.

PICKARD, A .S.; JOHNSON, J. A.; FARRIS, K. B. The Impact of Pharmacist Intervenctions on Health-Related Quality of Life. The Annals of Pharmacotehrapy. v. 33, n. 11, p. 1167-72, 1999.

RAINVILLE, E. C. Impact of pharmacist interventions on hospital readmissions for heart failure. Am J Health- Syst Pharm. v. 56, n. 13, p. 1339-1342, 1999.

ROMANO-LIEBER, N. S.; TEIXEIRA, J. J. V.; FARHAT, F. C. L. G.; et al. Revisão dos estudos de intervenção do farmacêutico no uso de medicamentos por pacientes idosos. Cad. Saúde Pública. v. 18, n.6, p. 1499-1507, 2002.

SANTORA, J.; KITRENOS, J. G.; GREEN, E. R. Pharmacist intervention program focused on i.v. ranitidine therapy. Am J Hosp Pharm. v. 47, n. 6, p. 1346-1349, 1990.

ZUBIOLI, A. O farmacêutico e a auto-medicação responsável. Pharmácia Brasileira. v. 3, n. 22, p. 23-26, 2000. 
Amaral, M. F. Z. J. et al./Revista Eletrônica de Farmácia Vol 5(1), 60-66, 2008.

WEIDLE, P.; BRADLEY, L.; GALLINA, J.; et al. Pharmaceutical care and related cost savings at a university hospital. Hospital Pharmacy. v. 34, n.1, p. 43-52, 1999. 